\title{
Coping Following Traumatic Brain Injury:The Need for Contextually Sensitive Assessment
}

\author{
Elizabeth Kendall \\ Center for Human Services, Griffith University, Australia \\ David Shum \\ Neuropsychology Unit, Griffith University, Australia \\ Brenda Lack, Susan Bull and Cameron Fee \\ Center for Human Services, Griffith University, Australia
}

\begin{abstract}
$\mathrm{P}$ sychosocial adjustment problems following traumatic brain injury ( $\mathrm{TBI}$ ) frequently present a major barrier for rehabilitation. The ability to cope has been linked with psychological well-being following stressful and traumatic events, but has been poorly examined in the area of TBI. In terms of conceptualisation, most coping research has adopted the Lazarus and Folkman (1984) dichotomy of coping, namely problem-focused and emotion-focused. Despite the popularity of this theory, recent conceptualisations of coping have suggested that other dimensions are equally important and require investigation in the TBI area. However, measurement of coping continues to provide a major barrier for research in this area, particularly given the potential difficulties associated with self-report data in people with TBI. The current study used a contextually sensitive assessment technique to test current conceptualisations of coping in the TBI population. Specifically, the study examined coping strategies and styles in response to four stressful video-based scenarios. Rather than using a self-report questionnaire to assess predetermined coping strategies, participants spontaneously provided their own coping strategies, which were then coded into distinct coping strategies. The strategies were categorised into four groups according to their focus (emotion or problem) and approach (active or passive). Both the number and type of coping strategies differed across situations, providing support for the use of a contextually sensitive measurement technique. Further, the theoretically expected pattern of relationships was found between coping types and outcomes. However, these relationships differed across situations and over time, confirming suggestions that coping efficacy may differ depending on the demands of the situation and that chronic situations such as TBI may have an impact on coping style over time.
\end{abstract}

Individuals who have sustained a traumatic brain injury (TBI) are likely to face significant difficulties in terms of their psychosocial adjustment (Finset \& Andersson, 2000). These individuals have to deal with the devastating consequences of their injury as well as many years of therapeutic demands. At the same time, they have to maintain emotional balance, a positive perception of themselves, sound relationships with their family and friends, and tolerance to an uncertain future. Although these complex adjustments necessitate advanced coping skills, little is known about

Address for correspondence: Elizabeth Kendall, Center for Human Services, Faculty of Health, Griffith University, Meadowbrook QLD 4131, Australia. 
coping in the TBI population (Kendall \& Terry, 1996). Unfortunately, research in this area is limited by difficulties associated with the conceptualisation of coping and the assessment methods that are currently employed. Thus, the purpose of the current research was to examine coping in the TBI population using a current conceptualisation of coping and a contextually sensitive assessment technique.

The ability to cope with "external or internal demands that are appraised as taxing or exceeding ones resources" (Lazarus \& Folkman, 1984, p. 141) has been linked to psychological wellbeing following stressful and traumatic events. In terms of conceptualisation, most coping research has adopted the Lazarus and Folkman (1984) dichotomy of coping, dividing coping efforts into those that are problem-focused and those that are emotion-focused. According to the theory, the primary aim of problem-focused coping is to actively confront a stressful event, either by altering the situation (environment-directed) or by acquiring the information or skills required to manage the situation (self-directed). In contrast, emotion-focused coping is a palliative response that aims to minimise the negative emotional impact of the event.

Although coping research has burgeoned over the last decade, little is known about coping among people who have sustained serious injuries or disabilities (Kendall, 1998). In the TBI area, coping research has yielded inconclusive results that do not clearly concur with the mainstream literature. For instance, studies of coping in the general population have tended to employ structured questionnaires to assess coping and have found that problem-focused coping is more effective than emotion-focused coping (Conway \& Terry, 1992; Felton \& Revenson, 1984). Similarly, in the TBI area, questionnaire studies have found that better outcomes were associated with lower levels of emotion-focused coping (e.g. Kendall, 1998) and that problem-focused coping strategies, including problem-solving, cognitive reappraisal and support seeking, were associated with positive outcomes (e.g. Moore, Stambrook \& Peters, 1989).

However, some researchers and clinicians have suggested that, over time, problem-focused coping may become less effective in response to a chronic condition such as TBI (Hinkeldey \& Corrigan, 1990). Although problem-focused coping might be an effective response in the short-term, many difficulties associated with permanent disability are likely to be resistant to such efforts. Thus, as time progresses, emotion- focused coping may be more effective than problem-solving in the TBI population. Indeed, Wineman, Durand and Steiner (1994) found that the use of problem-solving was not common among people with traumatic injuries and physical disabilities. Instead, these participants tended to rely on a combination of emotional avoidance and support seeking, indicating that the distinction between problem-focused and emotionfocused coping may be too simplistic in these complex populations.

Qualitative studies have also confirmed that people with serious disabilities tend to use a complex set of coping strategies, but that their coping style (i.e. their overall pattern of coping strategy use) is often skewed towards the emotion-focused end of the continuum. Specifically, Willer and his associates (Willer, Allen, Durnan, \& Ferry, 1990; Willer, Allen, Liss, \& Zicht, 1991) found that people with serious disabilities relied on a large range of strategies, such as avoiding the situation, suppressing emotions, trying to understand everything realistically and seeking support. Other less frequently nominated strategies that were considered important included maintaining a sense of humour, being optimistic and praying. Problemsolving was nominated by these individuals as a useful coping strategy, but only in the context of developing strategies or aids to compensate for disabilities, seeking rehabilitation or assistance and reallocating responsibility for tasks in the home.

Some evidence has suggested that these unclear findings could be attributed to differing conceptualisations of coping and the method by which coping is assessed. Specifically, the success of particular coping strategies may differ depending on the type of stressor that has precipitated the coping efforts. Failure to account for this situational context will weaken research and result in mixed findings (Kendall \& Terry, 1996). In addition, recent literature has indicated that there may be additional dimensions of coping that are important in determining the relative effectiveness of coping strategies. In this regard, some researchers have suggested that the effectiveness of coping depends on whether the strategy is active or passive in its approach to a stressful event (Finset \& Andersson, 2000). Finally, research has suggested that there is a need to examine aspects of the total coping style, particularly the flexibility and range of the coping repertiore (Schwartz \& Daltroy, 1999).

In relation to the context of coping, it has been suggested that both problem-focused and emotion-focused coping responses are likely to 
be used in most stressful encounters (Lazarus, 1990). However, the extent to which each strategy is successful is likely to be determined by the nature of that event (Lazarus, 1993). Indeed, Lazarus (1993) noted that a poor fit between the demands of the event and the focus of coping might not only be ineffective, but could actually be counterproductive in terms of psychosocial well-being. Despite the contextual specificity of coping, a great deal of research has required individuals to indicate how they cope with their illness, disability or injury as a unitary stressor (Kendall \& Terry, 1996). This approach does not address the fact that adjustment is likely to be influenced by the chronic strains and demands that accompany a stressful event rather than by the traumatic event itself (Lazarus, 1990). Given the fact that major stressful events, such as TBI, are likely to interfere with many life domains, the consideration of life events as unitary stressors has severely limited the theoretical and practical utility of many research findings (Somerfield \& Curbow, 1992). Thus, in assessing coping, it is important to acknowledge the contextual complexity of traumatic disability (Kendall \& Terry, 1996).

To overcome this difficulty, it is essential for studies to focus on the particular problems individuals with traumatic disabilities are most likely to experience rather than on the disability itself as a single stressful event. These daily problems provide a broad but consistent frame of reference within which individuals' coping efforts can be identified (Kendall \& Terry, 1996). Several studies have used qualitative methodologies to identify the daily stressors that are most likely to impact on people with TBI (e.g. Willer et al., 1990; Karlovits \& McColl, 1999). By assessing coping in the context of these multiple stressors, the subtle changes in the efficacy of particular types of coping strategies can be examined across situations, possibly accounting for discrepant findings in previous research.

In relation to the conceptualisation of coping, it may be necessary to distinguish between active and passive coping as well as problem-focused and emotion-focused strategies (Westman \& Shirom, 1995). For instance, problem-focused coping can involve active attempts to solve problems or passive efforts to alter one's interpretation of a problem situation (Terry \& Hynes, 1998). Similarly, emotion-focused strategies can range from passive, avoidant methods, such as suppression, wishful thinking, and distraction, to more active approach-oriented methods of emotional control, attempting to understand one's emotions and seeking emotional support. In some situations, active problem-focused coping might be maladaptive whereas passive problem-focused coping (e.g. problem redefinition) might be adaptive. The opposite pattern might be expected in response to other situations. It is also possible that active emotion-focused strategies (e.g. emotional release through support) could be useful in response to some stressors, even if passive emotion-focused strategies are maladaptive.

The impact of active and passive coping on psychosocial adjustment has not been adequately examined to date, particularly in the TBI area where coping research has not yet reached a high level of sophistication. In examining the activepassive dimension of coping, Terry and Hynes (1998) found that active problem-focused coping was not beneficial following an uncontrollable event (unsuccessful in-vitro fertilisation treatment) whereas passive problem-focused coping was associated with positive outcomes. Preliminary studies conducted in the TBI area have provided some support for the possibility that active and passive coping represent important dimensions of coping. For instance, one study found that passive emotion-focused coping was associated with depression in a TBI sample, and that this dimension of coping was more apparent than active emotion-focused coping or both active and passive problem-focused coping (Finset \& Andersson, 2000). In Krefting's (1989) ethnographic study, she found that individuals with TBI relied almost exclusively on passive emotionfocused strategies, such as denial and concealment, to manage the impact of TBI. Karlovits and McColl (1999) also used a qualitative and indirect methodology to identify coping strategies and found that people with TBI used a mix of passive and active strategies. However, these researchers noted that passive strategies were often applied successfully in order to avoid problem situations that could become further stressors.

Some researchers have speculated that access to a versatile and flexible repertoire of coping strategies (i.e. coping style) that can be altered in response to changing contextual demands is more important than consistently using a particular type of strategy (Pearlin \& Schooler, 1978). This notion is intuitively sensible as it acknowledges the dynamic nature of stressful encounters. However, in the TBI area, Moore and his associates (1989) found that individuals who reported using a wide range of coping strategies also reported a greater level of social and emotional disturbance than those who reported the frequent use of a small set of strategies. Similarly, Schwartz and Daltroy 
(1999) found that inconsistent coping over time was associated with neurological impairment and poor outcomes, whereas consistent use of a coping style was associated with improved outcomes. Clearly, there is a need to discriminate between adaptive (i.e. flexible) and maladaptive (i.e. haphazard) inconsistency. According to Kohlmann (1993), it may also be important to distinguish between rigidly passive or emotional coping styles, rigidly active or problem-solving coping styles, haphazard coping styles (i.e. high use of many strategies in all situations) and flexible coping styles (i.e. mixed use of a small set of strategies across situations). However, to date, little is known about the impact of coping styles and repertoires on psychosocial adjustment after stressful or traumatic life changes (Westman \& Shirom, 1995). In the context of TBI even less is known about the impact of coping style on adjustment.

Another important consideration in the study of coping among TBI populations is the fact that many studies have failed to confront the methodological problems associated with the use of selfreport inventories in the assessment of coping. Self-report measures of coping generally present respondents with a list of strategies and require them to indicate how frequently they have used each strategy over the last few weeks. It is unlikely that such techniques will assess actual coping and may lead to misinterpretations of the relationship between coping and psychosocial adjustment (Kendall, Shum, Halson, Bunning \& Teh, 1997). For instance, it has been found that the link between stated and actual behaviour is tenuous (Gosling, Oliver, Craik \& Robins, 1998), particularly among individuals who are cognitively impaired (Allen \& Ruff, 1990). Moreover, self-report measures of coping may simply assess the individual's ability to select the strategies that appear to be most socially appropriate or adaptive. In a similar vein, it is possible that completing a self-report inventory may provide respondents with cues about potential coping strategies, thus influencing their coping responses and biasing any research.

Finally, a major methodological concern associated with self-report measures of coping is that actual coping efforts may not be easily labelled by individuals or may be distorted by retrospective reports that are gained in the absence of contextual data. For instance, some strategies may be more amenable to verbalisation (e.g. problem-solving) than other strategies, such as avoidance, where the inability to accurately reflect on the situation is inherent in the strategy.
In this regard, Moore et al. (1989) identified a group of individuals with TBI who, despite the existence of severe injuries, reported an absence of both coping responses and psychosocial problems. Moore and his colleagues suggested that these individuals may have been denying the severity of their circumstances and, therefore, saw no need to implement coping strategies and reported good psychosocial outcomes. The fact that they may have been implementing passive emotion-focused coping could not be assessed using a self-report inventory.

Given the potentially important role of coping in psychological adjustment and rehabilitation, further investigation in the TBI population is warranted. Consequently, the major aim of this study is to examine the role of coping among people with TBI in response to the specific stressors they are likely to confront. In particular, the study will examine the relationships between adjustment and the four types of coping. These four types of coping are distinguished according to two dimensions, namely the focus of the coping (problem or emotion) and the approach of the coping (active or passive). Further, the study aims to examine the impact of flexibility and range of coping style on psychosocial outcome. On the basis of existing research, it is expected that there will be a relationship between active problem-focused coping and positive outcomes. There will also be a relationship between passive emotion-focused coping and negative outcomes. However, there may be a change in this pattern as time since injury increases. Specifically, active problem-focused coping may become less frequently nominated and less effective over time.

In examining these research questions, the study will also address the methodological problems associated with the assessment of coping. In particular, the study will develop and trial a contextually valid semi-qualitative technique that should be sufficiently sensitive to assess coping efforts across a range of situations. Based on the transactional theory of stress and coping put forward by Lazarus and Folkman (1984), it is expected that coping will differ depending on the characteristics of the situation that triggered the coping response.

\section{Method}

\section{Participants}

Participants in the study were 24 individuals (6 female and 18 male) who had sustained TBI at least 1 year previously but within the last 15 years $(M=7.75, S D=4.55)$. The ages of the partici- 
pants ranged from 20 to $51(M=33.96, S D=$ $9.68)$ and their level of education ranged from 9 to 13 years $(M=11.38, S D=1.24)$. Twenty of these individuals sustained a closed head injury as a result of a motor-vehicle accident and the remaining four from falls, an industrial accident, and a sporting accident. All except one participant had a GCS score of 8 or less at the time of admission to hospital and their duration of unconsciousness ranged from 1 to 180 days. All participants were recruited from a non-profit community organisation that provides information and support to individuals with brain injury. The participants took part in the study voluntarily by responding to an advertisement of the study in the newsletter of the organisation.

\section{Materials and Apparatus}

A semi-structured interview schedule was used to collect background information and the level of psychological adjustment. Adjustment was defined as acceptance of disability, self-esteem and affect and was measured using validated scales that have been commonly used in the TBI area (Kendall, 1998). To assess perceived stress and coping, four video vignettes were produced.

Acceptance of disability. This measure consisted of eight items adapted from Linkowski's (1971) Acceptance of Disability scale (Cronbach's alpha $=0.83)$. The wording of these questions was modified slightly for use in the traumatic brain injury population. These items were designed to assess positive acceptance of the injury and its associated limitations, and feelings of worth despite the effects of the injury. Example items included "I have a hard time adjusting to the limitations of my injury" and "Because of my injury, I miss the things I like to do most". Participants rated their agreement with each item on a 5-point Likert scale ranging from "strongly agree" to "strongly disagree". Negatively worded items were recoded so that higher scores indicated a greater level of acceptance.

Self-esteem. Rosenberg's (1965) 10-item Selfesteem Scale was used to assess the participants' overall sense of being capable, worthwhile, and competent $($ Cronbach's alpha $=0.81)$. Example items included "I feel that I have a number of good qualities" and "I am able to do things as well as most other people". Participants were asked to rate each item on a 5-point Likert scale ranging from "strongly disagree" to "strongly agree" and negatively worded items were recoded so that higher scores indicated a higher level of self-esteem.
Affect. Participants' positive and negative mood states were measured using the 10-item Bradburn Affect Balance Scale (1969). The scale required participants to indicate whether or not they had experienced five positive emotions and five negative emotions during the past six months, enabling the calculation of a total positive affect score and a total negative affect score. Higher scores indicated a higher level of each affect. The Cronbach's alpha for the five positive and the five negative questions were 0.72 and 0.64 respectively.

Video Vignettes. Four video vignettes were used to assess the level of perceived stress associated with different situations and the coping strategies that were generated in response to each situation. Each of the problem scenarios was approximately 1.5 to 2 minutes long. The four stressful scenarios used in the current study included (1) the inability to obtain a driving license, (2) discrimination at a job interview, (3) an unsuccessful social interaction, and (4) a memory-related problem. The themes of the scenarios were developed with the help of a focus group of five individuals with TBI. These themes were considered by this group to be common stressful situations faced by individuals with TBI. Such problems have also been identified through focus groups and qualitative interviews (e.g. Willer et al., 1990) as the most significant problems experienced by both single and married men with traumatic disabilities. As the majority of people with TBI are young men, these four common problem situations were considered appropriate for use in the current study.

All four scenarios were based on a central character named "Paul" who has sustained a traumatic brain injury at least a year ago. In the first scenario, Paul was told that he had to wait another month before he could obtain medical clearance to reapply for his driver's license. In the second scenario, Paul was refused a job on the ground that "he would be a bit of a risk in the job" due to his brain injury. In the third scenario, Paul was rejected by a female friend after a number of attempts to organise a date. In the last scenario, Paul was unable to remember whether he had borrowed money from a friend.

\section{Procedure}

All participants were assessed individually, either at their home or at Griffith University. The semistructured interview was always administered prior to the video vignettes, with a break between them. On average, each assessment session lasted about 1.5 hours, after which participants were debriefed about the scenarios and the purpose of 
the study. After completing the structured interview, the four vignettes were played and were repeated if necessary or requested. Following each vignette, participants were asked to briefly describe what had happened in the vignette to ensure their understanding of the situation. They were also asked to identify whether or not they had experienced a similar problem and, if so, how recently. To increase the salience of these memories, participants were asked to describe their own problem situations briefly. Once this process had been completed, participants were asked to rate how stressful they perceived each situation to be, irrespective of whether it had happened to them or if it was to happen in the future. This rating was made using a 10-point scale ranging from "not stressful at all" to "extremely stressful" so that a higher score indicated a higher level of perceived stress.

Finally, participants were asked to report how they would (or did) cope with each situation. Prompts (e.g. "how else would you cope?") were used to elicit as much information about coping responses as possible and exhaust each individual's coping repertoire. However, care was taken not to lead participants into reporting particular types of coping strategies. For instance, questions such as "how would you solve this situation" were avoided as this clearly implies the use of problem-focused coping. Similarly, questions such as "what would you do?" denote behavioural rather than cognitive strategies.

Participants' responses to the video vignettes were audio-taped for content analysis. Coping responses were analysed by two independent coders. The coders independently categorised all the coping responses given by participants into distinct strategies and inter-rater reliability was calculated for each scenario. Any disagreements about the number and type of coping responses reported were resolved by discussion between the two coders with reference to the definitions of coping strategies. If agreement could not be reached, another coder was consulted. Coders then categorised the coping responses according to whether or not they represented an active or passive strategy and whether the focus of coping was the problem or the emotions, creating four coping strategies. Active coping was defined as proactive attempts to alter either the problem or the emotions whereas passive coping was defined as strategies that were reactive or indirect. The score for each coping strategy was the frequency with which that type of strategy was nominated by participants, with higher scores indicating greater frequency.
The responses were also coded for flexibility of coping style based on the method used by Westman and Shirom (1995). Specifically, flexibility represented the extent to which strategies were repeated in response to the four different problem situations. Two flexibility scores were used, one representing general flexibility of coping style irrespective of the types of strategy being used and another representing the flexibility within each specific strategy type. General flexibility was operationalised by counting any strategy that was repeated in response to a subsequent scenario. Thus, if a participant used three strategies in response to the first scenario and repeated two of those strategies in response to the second scenario, the general flexibility score would be two. Thus, higher scores indicated less flexibility in the pattern of coping across scenarios (i.e. a more rigid style of coping). The maximum possible score for general flexibility would be 12 , indicating that all four coping strategies were repeated in all four scenarios.

In contrast, specific flexibility was operationalised as the repetition rate across scenarios for each type of coping, providing four scores one for each category of coping. This score was simply the number of scenarios in which that coping strategy was used (i.e. possible range of none to four), with a higher score indicating a more rigid reliance on that particular strategy. Thus, it was possible to obtain a measure of how rigidly each type of coping was applied across scenarios, a factor that could be obscured within the general flexibility score. For instance, it would be possible for a participant to rigidly rely on one type of coping, but vary his or her use of other types of coping leading to a low score on general flexibility (i.e. highly flexible). However, it is likely that this rigid reliance on one particular type of strategies, irrespective of other coping efforts, may have either a detrimental or beneficial impact on adjustment.

\section{Results}

\section{Perceived Level of Stress}

The average level of perceived stress for each of the four scenarios was above five, suggesting that they were perceived to be at least moderately stressful by the participants. The scenario perceived to be most stressful was the second scenario $(M=6.41, S D=2.43)$ where the central character was denied a job because of his brain injury. The next most stressful scenario was the first scenario $(M=6.00, S D=2.67)$ where the central character was told that he could not 
reapply for his driver's license. These scenarios were followed by the third scenario $(M=5.87, S D$ $=2.47$ ) where the central character failed to arrange a date successfully, and the fourth scenario $(M=5.20, S D=2.46)$ where he could not remember borrowing money from a friend. Nevertheless, a one-way repeated-measures ANOVA indicated that perceived stress did not differ significantly across the four situations.

\section{Number and Types of Coping Strategies}

In terms of number of strategies reported, interrater reliability was $100 \%$ for Scenario $1,98 \%$ for Scenario 2, 95\% for Scenario 3, and $100 \%$ for Scenario 4. A total of 303 coping strategies was generated by participants across all four scenarios. On average, participants generated 12.62 coping strategies in response to the four scenarios $(S D=3.30$; range from 7 to 19$)$. The most strategies were generated in response to the most stressful work-related scenario (91 strategies, $M=3.83$ strategies per participant, $S D=1.52$ ). The scenario concerning rejection by a girlfriend generated 81 strategies $(M=3.37$ strategies per participant, $S D=1.51$ ) and the driving scenario generated 71 strategies $(M=2.91$ strategies per participant, $S D=1.47$ ). Finally, the scenario concerning failure to remember borrowed money generated 60 strategies $(M=2.50$ strategies per participant, $S D=1.25$ ). A repeated-measures ANOVA indicated that the number of strategies generated in response to the four scenarios was significantly different $(F(3,69)=5.76 ; p<.001)$. Paired comparisons revealed that the number of strategies generated in response to the workrelated scenario was significantly greater than that generated for the driving $(t(1,23)=2.34$; $p<.05)$ and borrowed money scenarios $(t(1,23)=4.76 ; p<.001)$, but not significantly greater than the scenario concerning rejection by a girlfriend. The rejection scenario was associated with significantly more strategies than the borrowed money scenario $(t(1,23)=2.32 ; p<$ $.05)$. Despite similarities in the pattern of perceived stress and the number of strategies generated across the scenarios, the correlation cosefficients between these variables did not reach significance.

Within the data generated by participants, eight distinct coping responses were identified. Inter-coder agreement regarding the identification and coding of these responses was high for each scenario $(89 \%, 85 \%, 93 \%$ and $96 \%$ respectively). The most frequently nominated response was labeled instrumental action and was nominated 104 times by the total sample $(M=4.33, S D$
$=2.55)$. This response involved deliberate attempts to alter the problem situation, such as making a plan, taking affirmative steps, finding out further information or learning new skills. Coders categorised these responses as an active problem-focused strategy. The next most frequently nominated response (72 nominations, $M=3.00, S D=1.69$ ) involved attempts to accept the situation or make light of the problem. According to the coders, these responses represented a passive problem-focused strategy. These responses were followed in frequency by expressions of anger (57 nominations, $M=2.38, S D=$ 2.20), which involved statements of abuse or physical violence. Some difficulty was encountered in categorising these responses as, in a few cases, the anger represented an attempt to bully or frighten another person into providing the desired outcome (i.e. problem-focused coping). However, on closer inspection, these responses also represented attempts to avoid the actual problem (i.e. discrimination or rejection), focusing instead on their emotional reaction to the undesired outcome. The majority of responses $(n=49)$ involved attempts to obliterate the negative emotional impact of the situation without any direct reference to the problem (e.g. "punch walls" or "hit someone to get out my frustration"). Consequently, these responses were categorised as a passive emotion-focused strategy.

Three response types that were nominated less frequently included seeking support to problem solve ( 25 nominations, $M=1.04, S D=1.04$ ), exercising caution or control (18 nominations, $M=.75, S D=.94$ ), and avoidance (13 nominations, $M=.54, S D=.78)$. Support seeking involved direct attempts to mobilise, obtain or enlist the support and assistance of another person or organisation to solve a problem and was coded as an active problem-focused strategy. Although the mobilisation of social support can be an emotional exercise, the statements clearly indicated that the purpose of support was to provide an external source of guidance and practical help rather than assisting with the management of emotions. In contrast, exercising caution and control involved directly managing one's emotions to avoid doing or saying something that would cause further damage. Consequently, this strategy was coded as an active emotion-focused strategy. Avoidance involved obvious attempts to forget, deny or obliterate the negative consequences of a problem, usually through drinking, activity, or moving away. Avoidance was coded as a passive emotion-focused strategy. Finally, two responses that were nominated infrequently involved 
attempts to stay positive ( 9 nominations, $M=.38$, $S D=.71)$ and expressions of self-blame (5 nominations, $M=.21, S D=.41$ ). Staying positive was coded as an active emotion-focused strategy that was similar to looking on the bright side, remembering that things will come good one day and keeping one's spirits up. In contrast, self-blame involved severe recriminations against oneself, blaming oneself for everything and seeking to emotionally or physically punish oneself and was coded as a passive emotion-focused strategy.

After the responses were categorised according to both the focus and approach, active problem-focused strategies were the most common (129 nominations, $M=5.37, S D=3.00$ ), followed by passive emotion-focused strategies (75 nominations, $M=3.12, S D=2.62$ ). Passive problem-focused strategies were the next most commonly nominated strategies (72 nominations,
$M=3.00, S D=1.69)$ and active emotion-focused strategies were nominated least frequently (27 nominations, $M=1.12, S D=1.34$ ). As expected, repeated-measures ANOVA indicated that the mean number of strategies nominated in each category differed significantly $(F(3,69)=13.63 ; p<.0001)$. Table 1 shows some example responses and how they were categorised to form the four coping strategies.

\section{Use of Coping Categories Across Scenarios}

By examining strategy use across scenarios, it was possible to determine whether or not particular types of strategies were more likely to be generated in response to different stressors. Table 2 shows the frequencies, means and standard deviations of the nominated strategies across the sce-

\section{TABLE}

Examples of Coping Responses and the Categorisation into Four Coping Strategies

Example Responses

Coding Label and Definition

Coping Strategy

Instrumental Action ( $n=104)$

"Always have a notebook in future and write everything down"

"Talk through it - convince him that

I'm the best person for the job"

Deliberate attempts

to alter the problem

"Ask a friend if she remembers me borrowing the money"

Seeking Support $(n=25)$

Active Problem

"Call the authorities for advice - that is discrimination!"

Attempts to enlist assistance to solve the problem

"I'd just have to accept the wait and be patient"

Acceptance $(n=72)$

"If it is not meant to be, that's OK - just have to go on"

Attempts to accept

or make light of the problem

Passive Problem

$(n=72)$

Exercising Caution ( $n=18$ )

"I would control my feelings - not show them I'm upset"

"I'd try to have no reaction - there's no use crying about it"

Attempts to avoid doing or saying something emotional

"Something better will always come along - keep smiling"

"Remind yourself that one day it will all be OK"

Staying Positive $(n=9)$

Active Emotion $(n=27)$

Attempts to look on the bright side and feel good

"I'd try to do other things to keep my mind off it"

"Get out of there - walk — ride my bike like mad"

Avoidance $(n=13)$

Attempts to avoid the emotions associated with a problem

"I'd get so angry - typical bloody female

- she doesn't understand"

"I'd say stuff them all - I'd yell at them and maybe punch things"

Expression of Anger ( $n=57$ )

Attempts to avoid negative impact through expression

Passive Emotion $(n=75)$

"It'd be my fault - I probably didn't deal with her well

- upset her"

Self-blame $(n=5)$

"I'd curse myself for being in that accident

- I'll never be good enough" 
narios. The nomination of active problem-focused strategies was not significantly different across the scenarios, being generated at equally high frequency irrespective of the situation. However, the nomination of active emotion-focused strategies did differ significantly across the four scenarios $(F(3,69)=5.01 ; p<.001)$ as did the nomination of passive emotion-focused strategies $(F(3,69)$ $=4.70 ; p<.01)$ and the nomination of passive problem-focused strategies $(F(3,69)=7.81$; $p<.0001)$. Specifically, active emotion-focused strategies were nominated significantly more often in response to the driving scenario than the rejection scenario $(t(1,23)=2.04 ; p<.05)$ and the borrowed money scenario $(t(1,23)=4.41$; $p<.001$ ), but not more often than the work-related scenario. Passive emotion-focused strategies were nominated significantly less often in response to the borrowed money scenario than both the workrelated scenario $(t(1,23)=3.39 ; p<.01)$ and the rejection scenario $(t(1,23)=2.46 ; p<.05)$, but not the driving scenario despite a trend towards significance. Finally, the nomination of passive problem-focused strategies was significantly less common in response to the borrowed money and driving scenarios than both the rejection scenario $(t(1,23)=2.69$ and 3.89 respectively; $p<.05)$ and the work-related scenario $(t(1,23)=3.49 ; p<.01$ and $2.32 ; p<.05$ respectively). Thus, although active problem-focused coping was nominated in response to all situations, additional coping efforts differed depending on the scenario. Passive emotion-focused coping was common in response to the situations concerning discrimination whereas passive problem-focused coping was common in situations of both discrimination and social rejection. Active emotion-focused coping occurred commonly in response to scenarios concerning a delay in the achievement of driving and workrelated goals.

\section{Coping Style}

Participants provided between three and seven distinct coping responses $(M=4.70, S D=1.04)$ to each scenario, with the majority of participants using at least three of the four types of coping $(M=3.04, S D=.90)$. Across the scenarios, general flexibility scores ranged from 2 to 9 $(M=4.54, S D=1.77)$, indicating that most participants repeated strategies several times in their total response. The specific flexibility score for each category of coping across the scenarios was significantly higher for active problem-focused coping $(M=2.88, S D=1.23)$ than for active emotion-focused coping $(t(1,23)=4.60 ; p<.001$, $M=.88, S D=.99)$ and passive emotion-focused coping $(t(1,23)=2.20 ; p<.05, M=1.88$, $S D=1.03$ ), indicating that this type of coping was used most rigidly across scenarios. Passive problem-focused coping ( $M=2.33, S D=1.13)$ was

\section{TABLE 2}

Means, Standard Deviations and Frequencies of Each Coping Strategy in Response to the Scenarios

\begin{tabular}{|c|c|c|c|c|c|}
\hline & $\begin{array}{c}\text { Active } \\
\text { Emotion-focused }\end{array}$ & $\begin{array}{c}\text { Active } \\
\text { Problem-focused }\end{array}$ & $\begin{array}{l}\text { Passive } \\
\text { Emotion-focused }\end{array}$ & $\begin{array}{c}\text { Passive } \\
\text { Problem-focused }\end{array}$ & Total \\
\hline \multicolumn{6}{|c|}{ Scenario 1: Driving License Delay } \\
\hline Mean & .71 & 1.37 & .71 & .46 & 2.91 \\
\hline$S D$ & .41 & 1.40 & .95 & .51 & 1.47 \\
\hline Frequency & 10.00 & 33.00 & 17.00 & 11.00 & 71.00 \\
\hline \multicolumn{6}{|c|}{ Scenario 2: Work-related Discrimination } \\
\hline Mean & .33 & 1.41 & 1.25 & .79 & 3.83 \\
\hline$S D$ & .56 & 1.25 & 1.15 & .72 & 1.55 \\
\hline Frequency & 8.00 & 34.00 & 30.00 & 19.00 & 91.00 \\
\hline \multicolumn{6}{|c|}{ Scenario 3: Social Rejection } \\
\hline Mean & .33 & .96 & .75 & 1.33 & 3.87 \\
\hline$S D$ & .56 & .95 & .99 & 1.00 & 1.52 \\
\hline Frequency & 8.00 & 23.00 & 18.00 & 32.00 & 81.00 \\
\hline \multicolumn{6}{|c|}{ Scenario 4: Failure to Recall Borrowed Money } \\
\hline Mean & N/A & 1.63 & .41 & .42 & 2.50 \\
\hline$S D$ & N/A & 1.06 & .77 & .58 & 1.25 \\
\hline Frequency & 1.00 & 39.00 & 10.00 & 10.00 & 60.00 \\
\hline \multicolumn{6}{|l|}{ Overall } \\
\hline Mean & 1.12 & 5.37 & 3.12 & 3.00 & 12.62 \\
\hline$S D$ & 1.39 & 3.00 & 2.62 & 1.69 & 3.30 \\
\hline Frequency & 27.00 & 129.00 & 75.00 & 72.00 & 303.00 \\
\hline
\end{tabular}


repeated across scenarios at a significantly greater rate than active emotion-focused coping $(t(1,23)$ $=3.55 ; p<.05)$.

\section{Relationships Between Psychological Adjustment and Coping}

Table 3 shows the distributions, means and standard deviations for the four demographic variables (age, gender, educational level and injury time) and the four adjustment variables (esteem, acceptance, positive affect and negative affect).

To examine the relationships between adjustment and coping strategies, correlation coefficients were calculated between the four measures of adjustment (viz., acceptance of disability, selfesteem, positive and negative affect), the four categories of coping strategies, time since injury and the demographic variables. All bivariate plots were examined to ensure that these correlations were not inflated or deflated by the effects of outliers. Only one outlier was identified but its removal did not alter the significance of the correlation coefficient. The correlations between the demographic and injury variables, coping strategies and adjustment variables are contained in Table 4.

Gender, age and educational level were unrelated to any of the coping variables. However, education level was negatively correlated with positive affect $(r=-.42 ; p<.05)$, indicating that those with higher levels of education tended to report lower levels of well-being.

Significant correlations emerged between self-esteem and the use of passive emotionfocused coping $(r=-.71 ; p<.001)$. The use of active problem-focused coping was also significantly associated with self-esteem $(r=.42 ; p<$ $.05)$. These correlations indicated that the use of active problem-focused coping was associated

\section{TABLE 3}

Means, Standard Deviations and Ranges of the Demographic and Adjustment Variables

\begin{tabular}{|c|c|c|c|}
\hline Variable & Mean & $S D$ & Range \\
\hline Age & 33.96 & 9.68 & $20-51$ \\
\hline Gender & - & - & $\begin{array}{r}\text { Female }=6 \\
\text { Male }=18\end{array}$ \\
\hline Education level (years) & 11.38 & 1.24 & 9-13 \\
\hline Years since injury & 7.75 & 4.55 & $1-15$ \\
\hline Self-esteem & 37.88 & 7.52 & $18-50$ \\
\hline Acceptance & 23.33 & 9.00 & $10-40$ \\
\hline Negative Affect & 6.67 & 1.40 & $5-9$ \\
\hline Positive Affect & 9.00 & 1.25 & $6-10$ \\
\hline
\end{tabular}

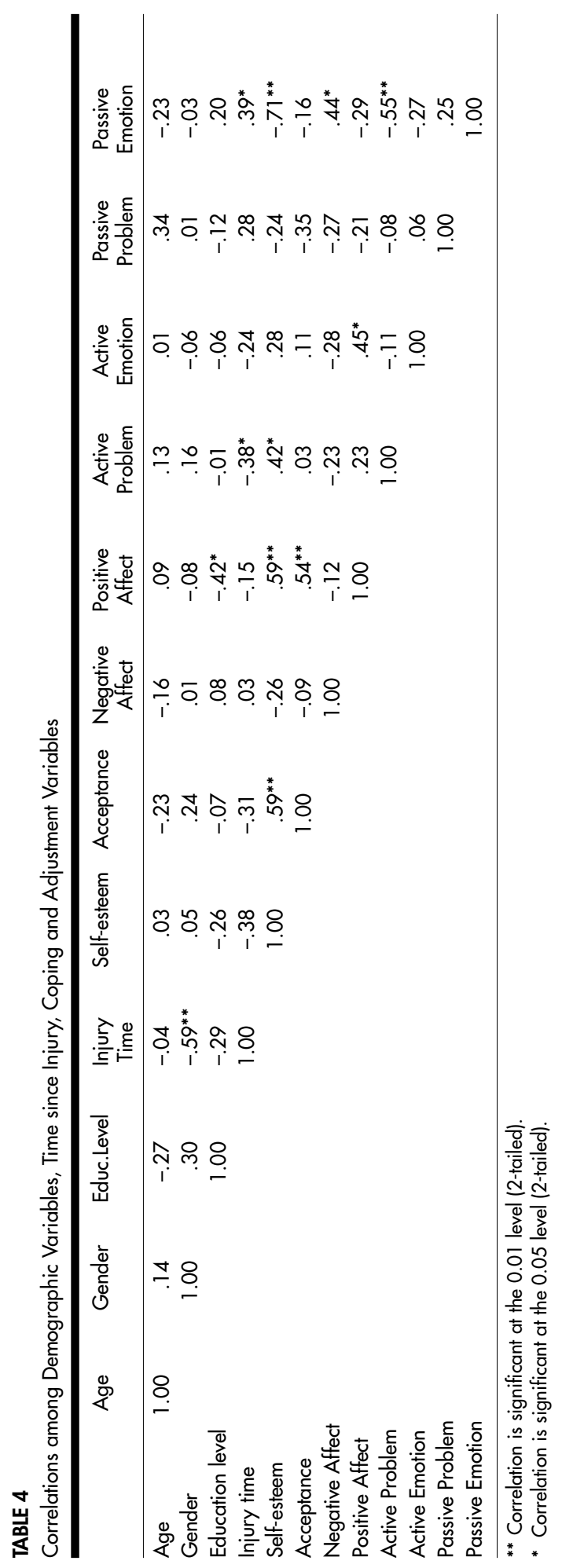


with higher levels of self-esteem whereas the use of passive emotion-focused coping was associated with lower levels of self-esteem. In relation to affect, significant correlations emerged between passive emotion-focused coping and negative affect $(r=.44 ; p<.05)$ and between active emotion-focused coping and positive affect $(r=.45 ; p<.05)$. Higher levels of negative emotion were associated with the use of passive emotion-focused coping whereas higher levels of positive emotion were associated with active emotion-focused coping efforts.

As expected, time since injury was significantly associated with the nomination of active problem-focused coping $(r=-.38 ; p<.05)$. As time since injury increased, the use of active problem-focused coping decreased. In contrast, the use of passive emotion-focused coping was positively associated with time since injury $(r=.39 ; p<.05)$, indicating that this type of coping increased over time. Time since injury was not associated with any adjustment variables. To further examine the change in relationships over time, partial correlations were conducted controlling for time since injury. After controlling for injury duration (see Table 5), the correlation between self-esteem and passive emotionfocused coping remained significant $(r=-.66$; $p<.001)$. Thus, the use of passive emotionfocused coping appeared to have a consistently negative relationship with self-esteem over time. Similarly, the relationship between positive affect and active emotion-focused coping $(r=.43$; $p<.05)$ remained significant. The remaining relationships were no longer significant after controlling for injury duration.

The efficacy of coping strategies across situations was examined by re-calculating the correlations for each scenario as shown in Table 6. There were no significant correlations between coping type and outcomes for the driving scenario. However, in response to the work-related scenario, significant correlations emerged between the use of passive problem-focused coping which was negatively associated with positive affect $(r=$ $-.34 ; p<.05$ ) indicating that greater use of passive problem-focused coping was associated with less positive affect. Higher levels of esteem were associated with less use of passive problemfocused and passive emotion-focused coping ( $r=$ -.34 and $-.49 ; p<.05$ respectively) but greater use of active problem-focused coping $(r=.41 ; \mathrm{p}$ $<.05)$. In response to the rejection scenario, greater use of passive emotion-focused coping was associated with higher levels of negative affect $(r=.38 ; p<.05)$ and lower levels of both positive affect $(r=-.39 ; p<.05)$ and esteem $(r=$ $-.62 ; p<.001)$. Finally, in response to the borrowed money scenario, esteem was positively correlated with active problem-focused coping $(r=.37 ; p<.05)$ but negatively correlated with both passive emotion-focused coping and passive problem-focused coping $(r=-.43$ and -.41 respectively; $p<.05$ ).

As shown in Table 7, general flexibility was not associated with any adjustment variables. When specific flexibility for each coping category was examined, a significant correlation was found between the repetitive use of active problem-focused coping and higher self-esteem $(r=.44 ; p<.05)$. Repetitive use of active emotion-focused coping was associated with higher levels of positive affect $(r=.44 ; p<.05)$. In contrast, repetitive use of passive emotion-focused coping was associated with higher levels of negative affect $(r=.45 ; p<.05)$ and lower levels of esteem $(r=-.56 ; p<.01)$.

\section{Discussion}

The current study examined coping strategies and styles in response to four stressful scenarios. Rather than using a self-report questionnaire to assess predetermined coping strategies, participants spontaneously provided their own responses to stressful scenarios. These responses were coded and categorised by two independent

\section{TABLE 5}

Partial Correlations among Coping and Adjustment Variables Controlling for Time since Injury

\begin{tabular}{lcccc} 
& Self-esteem & Acceptance & Negative Affect & Positive Affect \\
\hline Active Problem & .32 & -.10 & -.23 & .19 \\
Active Emotion & .22 & .04 & -.28 & $.43^{*}$ \\
Passive Problem & -.14 & -.28 & -.30 & -.17 \\
Passive Emotion & $-.66^{* *}$ & -.04 & .46 & -.26 \\
\hline
\end{tabular}

** Correlation is significant at the 0.01 level (2-tailed).

* Correlation is significant at the 0.05 level (2-tailed). 


\section{TABLE 6}

Correlations between Adjustment Variables and Coping Strategies for Four Scenarios

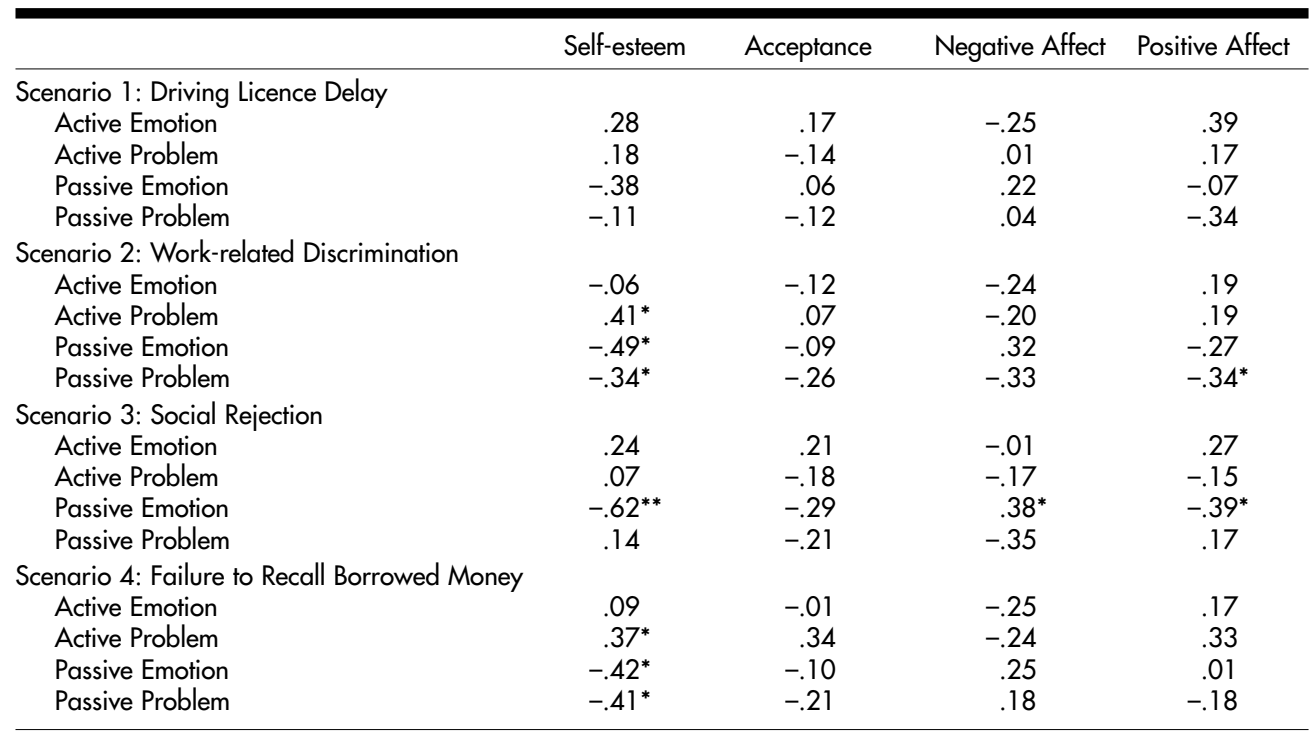

** Correlation is significant at the 0.01 level (2-tailed).

* Correlation is significant at the 0.05 level (2-tailed).

\section{TABLE 7}

Correlations between Adjustment Variables and Coping Flexibility

\begin{tabular}{lcccc} 
& Self-esteem & Acceptance & Negative Affect & Positive Affect \\
\hline General Flexibility & -.22 & -.10 & .01 & .04 \\
Rigid Active Emotion & .24 & .25 & -.28 & $.46^{*}$ \\
Rigid Active Problem & $.44^{*}$ & .18 & -.35 & .20 \\
Rigid Passive Problem & -.28 & -.26 & -.09 & -.31 \\
Rigid Passive Emotion & $-.56^{* *}$ & -.15 & $.45^{*}$ & -.10 \\
\hline
\end{tabular}

** Correlation is significant at the 0.01 level (2-tailed).

* Correlation is significant at the 0.05 level (2-tailed).

coders into four distinct coping strategies, according to their focus (emotion or problem) and approach (active or passive). There were several important findings of the current study. First, it was found that both the number and type of coping strategies differed significantly across situations, providing support for the use of a contextually sensitive measurement technique. Second, the expected pattern of significant relationships was found between coping types and outcomes. However, also as expected, these relationships differed across situations and over time. Finally, contrary to expectations, general flexibility was not associated with adjustment. However, the rigid use of active problem-focused coping in response to all situations was associated with high self-esteem. Surprisingly, the rigid use of active emotion-focused coping was also associated with better emotional outcomes, but as expected, the rigid use of passive emotionfocused coping was associated with both poor emotional outcomes and low self-esteem.

Active problem-focused coping was nominated most often and consistently across all the scenarios. Although other studies have found a similarly high level of active problem-focused coping among people with TBI, Karlovits and McColl (1999) noted that this high incidence could result from the fact that such strategies are consistent with rehabilitation goals. Accordingly, 
participants may perceive this type of strategy to be the most acceptable response irrespective of whether or not they would actually use such strategies. Nevertheless, both passive emotionfocused coping and passive problem-focused coping were nominated frequently, as found by other researchers (e.g. Karlovits \& McColl, 1999; Westman \& Shirom, 1995). Further, the high frequency with which 'socially unacceptable' strategies, such as abuse and aggression, were generated indicated that participants were not unduly swayed by social desirability.

In terms of the relationships between coping and adjustment, active problem-focused coping was generally associated with high self-esteem and positive affect whereas passive emotionfocused coping was associated with poor selfesteem and negative affect. As expected, passive emotion-focused coping was consistently associated with poor outcomes across all four scenarios. These findings are in accordance with the general coping literature. For instance, some researchers have found problem-focused coping to be the most effective coping strategy and emotionfocused coping to be the least effective strategy, regardless of the situation (Conway \& Terry, 1992; Felton \& Revenson, 1984).

However, some important differences emerged in the current study when the scenarios were examined independently. In accordance with current coping theory (Finney, Mitchell, Cronkite \& Moss, 1984), the most stressful scenario was associated with the greatest number of coping strategies, even though the relationship between stress and coping was not significant. Although active problem-focused coping was the most common type of coping across all situations, its presence was most evident in the scenario concerning the inability to remember borrowed money. Willer et al. (1990) also found that problem solving was regularly nominated by people with TBI, but most often in dealing with cognitive difficulties. The strategies nominated in response to this scenario were mostly aimed at improving the way in which one documented borrowed money or enlisting the help of friends who would accurately recall whether or not money had been borrowed.

Most participants indicated that they could actively manage this type of situation, supporting the notion that the perception of control is a significant predictor of the use of problem-solving (Lazarus, 1990). In accordance with coping theory, the use of problem-solving in response to such a controllable situation was associated with high levels of self-esteem whereas greater use of passive emotion-focused or passive problem-focused coping was associated with lower levels of selfesteem. In this regard, several researchers have found that the use of problem-solving strategies is associated with well-being in situations considered to be amenable to change but not in low control situations (Terry \& Hynes, 1998; Vitaliano, DeWolfe, Maiuro, Russo \& Katon, 1990). However, due to the correlational analysis used in this study, it is unclear which variable preceded the other. Specifically, it is equally likely that high self-esteem acted as a coping resource that enabled people to use active problem-focused coping (Terry, 1994).

More definitive conclusions can be drawn from the social rejection and work-related scenarios. Within their comments, most participants indicated that they believed they would have little control over these situations. However, the social rejection scenario appeared to be more manageable than the work-related scenario, which appeared to lead to a different style of coping. Participants recognised that they had to accept social rejection, but noted that they were capable of shifting their attention elsewhere in future, providing some measure of control. This scenario was associated with a high frequency of passive problem-focused coping. In contrast, the workrelated scenario generated the highest frequency of passive emotion-focused strategies, including strong expressions of anger and self-blame. In response to the frustration created by this scenario, participants indicated that they would "punch a wall", "tell the boss to get lost" or "abuse him". This scenario was clearly seen as an uncontrollable and unjust event that left participants feeling helpless. In both scenarios, the nomination of emotion-focused strategies was associated with poor levels of self-esteem. This pattern of results clearly demonstrates the link between perceived controllability, type of coping and outcome (Lazarus, 1990) and supports the existence of a relationship between high levels of stress or frustration and emotion-focused coping (Frederikson \& Dewe, 1996).

An important finding of the current study was that the nomination of passive emotion-focused coping increased as time since injury increased whereas the nomination of active problemfocused coping decreased over time. The negative relationship between passive emotion-focused coping and low self-esteem remained significant irrespective of time since injury, indicating that this was a pervasive relationship. In contrast, the beneficial impact of active problem-focused coping disappeared when time since injury was 
controlled and a significant relationship emerged between active emotion-focused coping and positive affect. This pattern of results indicates that over time, active problem-focused coping may become less effective, whereas active emotionfocused coping may become a useful strategy. A similar temporal pattern of coping was highlighted by Hinkeldey and Corrigan (1990). These researchers suggested that, given the permanence and severity of the changes created by TBI, active attempts to problem-solve may become frustrating, leading to feelings of helplessness and depression. Consequently, problem-solving attempts may be abandoned over time in favour of more emotional types of coping.

This coping pattern mirrors qualitative descriptions of the adjustment process following TBI and other chronic conditions. For example, Newsome and Kendall (1996) showed how an unexpected shift in life circumstances is likely to result in the persistent use of familiar strategies and continual attempts to restore the original circumstances. As Kendall and Buys (1998) noted, failure to restore one's situation is likely to result in intense emotions that overwhelm the individual and are unable to be managed effectively. However, over time, people with TBI may gradually integrate their new circumstances into their sense of self and manage their intense emotions more effectively (Yoshida, 1993). Clearly, if the adjustment process is to be fully understood and facilitated, there is a need to examine coping processes in a longitudinal manner, mapping efforts as they transpire during the course of a stressful event.

The ability to modify one's coping efforts, either during the course of a stressful event or in response to different events, has been considered to be a beneficial skill (Kohlmann, 1993). In contrast, studies in the TBI area have found that the use of a wide range of coping strategies was not beneficial, but that heavy reliance on a few problem-focused strategies was associated with good outcomes (Moore et al., 1989). Interestingly, in the current study, rigid use of active problemfocused coping or active emotion-focused coping was associated with positive outcomes whereas rigid reliance on passive emotion-focused coping across situations was associated with negative outcomes. These findings suggest that a great deal more research is needed to understand the role of coping style.

In this regard, it may be necessary to distinguish between indiscriminate or haphazard coping and responsive or flexible coping, a requirement that might best be achieved by mea- suring the perceived effectiveness of one's coping efforts (Cheng, 2001). Presumably, highly flexible coping that is not perceived or expected to be effective may represent haphazard coping. Indeed, a recent qualitative study conducted by Kendall and Kendall (2001) based on the published writings of individuals with TBI identified frequent references to periods of 'trial-and-error' coping. During this time, participants indicated that they did not know what to do and simply did as many different things as they could, hoping to learn from their failures. Thus, it is possible that the measurement of general flexibility used in the current study may have assessed this type of trial and error approach. Further, the flexibility measures were likely to be inherently confounded with the type of cognitive deficits that are commonly experienced by a TBI population. Consequently, future research in this area should provide a more thorough analysis of cognitive impairment that was not available in the current study.

It is also possible that conventional conceptualisations of coping are challenged in the context of TBI and other chronic disabling conditions. For instance, Wineman, Durand and McCulloch (1994) found that the factor structure of the Ways of Coping self-report questionnaire was not replicated in spinal cord injury and multiple sclerosis populations. Instead, the responses of these groups were more clearly represented by a threefactor structure consisting of cognitive reframing, emotional respite and direct assistance, each of which represented a mixture of traditional coping types. Finset and Andersson (2000) also found that participants with neurological impairment tended to display less differentiated coping styles than those without neurological impairment. In further support of this possibility, Wineman et al. (1994) noted that participants with TBI verbally reported using coping strategies that were not represented in the Ways of Coping questionnaire.

Using an indirect interview methodology, Karlovits and McColl (1999) identified eight common coping strategies. Although these strategies could be classified into the recognised conceptualisation of coping, Karlovits and McColl noted a qualitative difference between the purposes of these strategies and the accepted definitions of coping. Another qualitative study using written accounts of people with TBI (Kendall \& Kendall, 2001) found that 44 distinct coping strategies were described by people with TBI. By collapsing these strategies into a recognised typology of coping, important information was lost, particularly in relation to the differing time periods within which different strategies were 
used. Together, these findings indicate that there is a strong likelihood that the actual coping efforts of people with TBI may differ markedly from those of people without disabilities. Consequently, although the current study provided a more contextually valid measure of coping and avoided the difficulties associated with a pre-determined self-report inventory, grouping strategies according to recognised categories may have obscured important detail and, possibly, restricted the relationships between coping and outcome.

In addition to these limitations, serious problems were created by the small sample size, the large number of analyses and the absence of a longitudinal design. Nevertheless, the findings have confirmed the importance of assessing coping across situations. The study has significant clinical and rehabilitation implications in that it is necessary to assist people to tailor their coping efforts to the demands of the situation. Although it is important to assist people to effectively problem-solve, it is also important to assist them to recognise situations where they must manage emotions or alter their own perceptions. However, encouraging the indiscriminate use of many coping strategies is not beneficial for wellbeing. It may be most beneficial to assist people to analyse the demands of each situation, a skill that people with TBI may find extremely difficult (Kendall et al., 1997).

Future coping research may be most helpful if it identifies the relative effectiveness of strategies across the life of a stressful event. This analysis of processes and sequences will require longitudinal qualitative research and may benefit from a case study diary-based methodology that enables participants to describe in detail their responses to only those situations they have experienced. Leonard-Barton (1990) suggested that the case study is the most appropriate framework for studying processes and the links between processes and outcomes, particularly when those processes have not been thoroughly researched or are not clearly understood. The approach provides rich detail about a complex phenomenon and facilitates an understanding of the interactions between many subtle factors that may not emerge in quantitative studies. When combined with a longitudinal design, the case study diary is particularly useful as a means of tracing decision-making processes and incremental outcomes that may not be accessible otherwise, may be considered by participants to be "trivial" or could be easily forgotten (Elliot, 1997).

\section{Acknowledgments}

This paper was funded in part by the Motor Accident Insurance Commission of Queensland and the Centre for National Research on Disability and Rehabilitation Medicine. Sincere thanks are due to the people with traumatic brain injury who helped us to develop and test the video.

\section{References}

Allen, C.C. \& Ruff, R.M. (1990). Self-rating versus neuropsychological performance of moderate versus severe head-injured patients. Brain Injury, 4, 7-17.

Bradburn, N. (1969). The structure of psychological well-being. Chicago: Aldine.

Cheng, C. (2001). Assessing coping flexibility in reallife and laboratory settings: A multimethod approach. Journal of Personality and Social Psychology, 80, 814-833.

Conway, V.J. \& Terry, D.J. (1992). Appraised controllability as a moderator of the effectiveness of different coping strategies: A test of the goodness of fit hypothesis. Australian Journal of Psychology, 44, 1-7.

Elliot, H. (1997). The use of diaries in sociological research on health experience. Sociological Research Online, 2(2), 1-12.

Felton, B.J. \& Revenson, T.A. (1984). Coping with chronic illness: A study of controllability and the influence of coping strategies on psychological adjustment. Journal of Consulting and Clinical Psychology, 52, 343-353.

Finney, J.W., Mitchell, R.E., Cronkite, R.C. \& Moos, R. (1984). Methodological issues in estimating main and interactive effects: Examples from coping, social support and stress field. Journal of Health and Social Behavior, 25, 85-98.

Finset, A. \& Andersson, S. (2000). Coping strategies in patients with acquired brain injury: Relationships between coping, apathy depression and lesion location. Brain Injury, 14, 887-905.

Frederikson, L.G. \& Dewe, P.J. (1996). A longitudinal study of appraisal and coping using repeated measures of stressor, importance, frustration and coping response. Stress Medicine, 12, 81-91.

Gosling, S.D., Oliver, P.J., Craik, K.H. \& Robins, R.W. (1998). Do people know how they behave? Journal of Personality and Social Psychology, 74, 1337-1349.

Hinkeldey, N.S. \& Corrigan, J.D. (1990). The structure of head injured patients' neurobehavioral complaints: A preliminary study. Brain Injury, $4(2), 115-133$.

Karlovits, T. \& McColl, M.A. (1999). Coping with community reintegration after severe brain injury: A description of stresses and coping strategies. Brain Injury, 13, 845-861. 
Kendall, E. (1998). Coping and adjustment following closed head injury: A model for understanding individual differences and predicting outcomes. Unpublished Thesis. Brisbane: UQ.

Kendall, E. \& Buys, N. (1998). Models of psychosocial adjustment for rehabilitation counselling. The Journal of Rehabilitation, 64, 16-20

Kendall, E. \& Kendall, M. (2001). Written accounts of coping strategies among people with traumatic brain injury. Centre for Human Services Monograph. Brisbane: Griffith University.

Kendall, E. \& Terry, D. (1996). Psychosocial adjustment following closed head injury: A model for predicting outcome. Neuropsychological Rehabilitation, 6, 101-132.

Kendall, E., Shum, D., Halson, D., Bunning, S. \& Teh, M. (1997). The assessment of social problem solving skills following closed head injury. Journal of Head Trauma Rehabilitation, 12, 69-79

Kohlmann, C.W. (1993). Rigid and flexible models of coping. Anxiety Stress and Coping, 6, 107-123.

Krefting, L. (1989). Reintegration into the community after head injury: The results of an ethnographic study. The Occupational Therapy Journal of Research, 9, 67-83.

Lazarus, R. \& Folkman, S. (1984). Stress, appraisal and coping. NY: Springer.

Lazarus, R. (1990). Theory-based stress measurement. Psychological Inquiry, 1, 3-13.

Lazarus, R. (1993). Coping theory and research: Past, present future. Psychosomatic Medicine, 55, 234-247.

Leonard-Barton, D. (1990). A dual methodology for case studies: Synergistic use of a longitudinal single site with replicated multiple sites. Organization Science, 1(3), 248-266.

Linkowski, D.S., (1971). Acceptance of disability. Rehabilitation Counseling Bulletin, 14, 238-244.

Moore, A.D., Stambrook, M. \& Peters, L.C. (1989). Coping strategies and adjustment after closed head injury: A cluster analytical approach. Brain Injury, 3, 171- 175 .

Newsome, R. \& Kendall, E. (1996). Empowerment rehabilitation: An alternative to restoration. Australian Journal of Rehabilitation, 2, 71-85.
Pearlin, L.I. \& Schooler, C. (1978). The structure of coping. Journal of Health and Social Behavior, $12,2-21$.

Rosenberg, M. (1965). Society and the adolescent self image. New Jersey: Princeton University Press.

Schwartz, C.E. \& Daltroy, L.H. (1999). Learning from unreliability: The importance of inconsistency in coping dynamics, Social Science \& Medicine, 48, 619-633.

Somerfield, M. \& Curbow, B. (1992). Methodological issues and research strategies in the study of coping with cancer. Social Science Medicine, $34,1203-1216$

Terry, D.J. \& Hynes, G. (1998). Adjustment to a low control situation. Journal of Personality and Social Psychology, 74, 1078-1092.

Terry, D.J. (1994). The determinants of coping: The role of stable and situational factors. Journal of Personality and Social Psychology, 66, 895-910.

Vitaliano, P.P., DeWolfe, D.J., Maiuro, R.D., Russo, J. \& Katon, W. (1990). Appraised changeability of a stressor as a modifier of relationship between coping and depression. Journal of Personality and Social Psychology, 59, 582-592.

Westman, M. \& Shirom, A. (1995). Dimensions of coping behaviour: A proposed conceptual framework. Anxiety, Stress and Coping, 8, 87-100.

Willer, B., Allen, K., Durnan, M.C. \& Ferry, A. (1990). Problems and coping strategies of mothers, siblings and young adult males with traumatic brain injury. Canadian Journal of Rehabilitation, 3, 167-173.

Willer, B., Allen, K., Liss, M., \& Zicht, M. (1991). Problems and coping strategies of individuals with traumatic brain injury and their spouses. Archives of Physical Medicine and Rehabilitation, 72, 460-464.

Wineman, M.N., Durand, E.J., \& McCulloch, J.E. (1994). Examination of the factor structure of the ways of coping questionnaire with clinical populations. Nursing Research, 43, 268-273.

Wineman, M.N., Durand, E.J., \& Steiner, R.P. (1994). A comparative analysis of coping behaviors in persons with multiple sclerosis or a spinal cord injury. Research in Nursing and Health, 17, 185-194.

Yoshida, K.K. (1993). Reshaping of self: A pendular reconstruction of self and identity among adults with traumatic spinal cord injury. Sociology of Health and Illness, 15, 217-245. 